Georgia State University

ScholarWorks @ Georgia State University

\title{
A preliminary investigation of Acceptance and Commitment Therapy as a treatment for chronic skin picking
}

M. P. Twohig

S. C. Hayes

Akihiko Masuda

Georgia State University, amasuda@gsu.edu

Follow this and additional works at: https://scholarworks.gsu.edu/psych_facpub

Part of the Psychology Commons

\section{Recommended Citation}

Twohig, M. P.; Hayes, S. C.; and Masuda, Akihiko, "A preliminary investigation of Acceptance and Commitment Therapy as a treatment for chronic skin picking" (2006). Psychology Faculty Publications. 99.

https://scholarworks.gsu.edu/psych_facpub/99

This Article is brought to you for free and open access by the Department of Psychology at ScholarWorks@ Georgia State University. It has been accepted for inclusion in Psychology Faculty Publications by an authorized administrator of ScholarWorks @ Georgia State University. For more information, please contact scholarworks@gsu.edu. 
RUNNING HEAD: ACT for Skin Picking

A Preliminary Investigation of Acceptance and Commitment Therapy as a Treatment for Chronic Skin Picking

Michael P. Twohig

Steven C. Hayes

Akihiko Masuda

University of Nevada, Reno

Editorial correspondence should be addressed to: Michael Twohig

Department of Psychology

University of Nevada

Mail Stop 298

Reno, NV 89557-0062

e-mail: twohigm@unr.nevada.edu

phone: (775) 784-6828 X2011 


\begin{abstract}
The effectiveness of a deliberately limited version of Acceptance and Commitment Therapy for chronic skin picking was evaluated in a pair of multiple baseline across participants designs. Self monitoring of skin picking showed that four of the five participants reached near zero levels of picking by post-treatment, but these gains were not fully maintained for three of the four participants at follow-up. The findings of the self-reported skin picking were generally corroborated by ratings of photographs of the damaged areas and by ratings on a validated measure of skin picking severity. All participants rated the intervention as socially acceptable, and reductions were found on measures of anxiety, depression, and experiential avoidance for most participants as a result of the intervention. Results support the construction of more comprehensive ACT protocols for skin-picking.
\end{abstract}

Key Words: Acceptance; Acceptance and Commitment Therapy; ACT; Cognitive therapy; Skin picking; Treatment 
A Preliminary Investigation of Acceptance and Commitment Therapy as a Treatment for Chronic

\section{Skin Picking}

Skin picking is prevalent, disturbing, and difficult to treat. It occurs in just under $3 \%$ of college students (Bohne, Wilhelm, Keuthen, Baer, \& Jenike, 2002; Teng, Woods, Twohig, \& Marcks, 2002) and is more prevalent in females (Teng et al., 2002). Physically, skin picking can produce scarring and skin infections, and in rare cases is nearly fatal (O’Sullivan, Phillips, Keuthen, \& Wilhelm, 1998). Psychological effects can include guilt, shame, and embarrassment from the resulting damage (Bohne et al., 2002).

There is little information regarding best practices for clinicians treating this problem. While several case reports exist, there are only two controlled treatment studies (Teng, Woods, \& Twohig, in press; Twohig \& Woods, 2001), and both evaluated the effectiveness of simplified habit reversal (SHR). In the first of these studies, the effect of SHR was evaluated in a multiple baseline design with two adult brothers who skin picked (Twohig \& Woods, 2001). Participants reduced their self-monitored picking, although both showed some picking at the end of treatment. Gains were maintained in one participant at three-month follow-up, but the other lost over half of the gains. Both showed objective improvements on skin ratings at post-treatment, and some loss of that improvement at follow-up. The second investigation (Teng et al., in press) utilized both a SHR and waitlist control group (N=19). Self-monitored picking decreased by $77 \%$ from pre-treatment to post-treatment in the SHR group and averaged about 6 picks per day after treatment. No significant decreases in picking were found for the wait-list control group. Treatment gains were maintained at a three-month follow-up for the SHR group. No participants reached zero picking levels at post-treatment, however one did at follow-up. Average skin damage ratings at follow-up were improved but still elevated (2.08 on a three point scale for the 
control group; 1.86 for the SHR group). Thus, as Teng et al. noted "treatment gains, although substantial, were not complete” (p. 10). Continued research on skin picking treatment seems necessary.

One possible approach is based on the idea that body focused repetitive behaviors (BFRB) can serve different behavioral functions (du Toit, Kradenburg, Niehaus, \& Stein, 2001; Twohig \& Woods, 2004). Some BFRB's seem to be performed outside of the individual's verbal awareness, while others have a more verbal, anticipatory, and obsessive compulsive-like presentation. In the trichotillomania literature these two types are termed "automatic" and “focused,” respectively (Christenson \& Mansueto, 1999); this distinction seems applicable to skin picking as well. It is presumed that some BFRB's serve an emotional avoidance function. That means, for some, the picking is partially done to reduce an aversive private event. For example, in trichotillomania high emotional avoiders have more frequent and intense urges to pull, are less able to control their urges, and experience more pulling-related distress than persons who are not experientially avoidant (Begotka, Woods, \& Wetterneck, 2004). Emotional avoidance functions seem particularly likely to occur with focused skin picking, which is associated with feelings of tension when resisting picking or a sense that picking cannot stop until things are “right.”

Acceptance and Commitment Therapy (ACT, said as one word - not initials; Hayes, Strosahl, \& Wilson, 1999) is a behavior analytic psychotherapy that specifically targets the behavioral rigidity (restricted behavioral repertoire) that can come from experiential avoidance (an unwillingness to experience certain thoughts and feelings and engaging in behaviors to reduce them even when attempts are themselves harmful) and the dominance of verbal rules over direct contact with contingencies (what is termed “cognitive fusion”). ACT assumes that verbally 
based processes often need to be targeted in addition to direct behavior change. ACT teaches clients to notice their thoughts and feelings as one might notice an external event, so that the process of thinking becomes more evident and less believable. ACT uses a variety of methods to reduce the function of that verbalization when it occurs (what is called "cognitive defusion" in $\mathrm{ACT})$.

Similarly, ACT targets attempts to get rid of private events such as anxiety, fear, or urges out of the view that such escape and avoidance behaviors will only increase their saliency (and perhaps even their frequency). Instead, acceptance and mindfulness methods are used to teach the person how to more fully experience emotions, while linking actual overt behavior to appetitive valued ends rather than to avoidance.

The possibility that some BFRB's may have experiential avoidance functions has led researchers to combine ACT with traditional behavioral interventions such as SHR. Two studies have demonstrated that ACT plus SHR is effective in reducing trichotillomania (Twohig \& Woods, 2004; Woods, Wetterneck, \& Flessner, in press). In this study, the effectiveness of an eight session ACT protocol for skin picking that specifically avoided components of SHR was evaluated in a multiple baseline design to see if ACT alone can reduce skin picking in typically developing adults. If this protocol is shown to impact skin picking more comprehensive packages can then be constructed with the knowledge that the added ACT alone can reduce skin picking and presumably other BFRB's.

\section{Method}

\section{Participants}

Participants were recruited through postings on the local university campus, orally in undergraduate psychology classes, and through advertisements in the local newspaper. Six 
individuals enrolled in the study. One dropped out during baseline because she moved to a different state, leaving five participants. All individuals met criteria for chronic skin picking as defined by previous skin picking research (Teng et al., in press). All participants were Caucasian women, and no participants reported the use of any psychotropic medications within the previous 4 weeks (additional participant data are reported in Table 1).

Participant 2 also met DSM-IV-TR (APA, 2000) criteria for trichotillomania. She reported that when she felt the urge to pick she would either skin pick or hair pull; she stated that the two behaviors served the same psychological function. When she picked at her arms she reported sometimes removing "bulbs;" it is possible that she was removing hair follicles from her arms. The client was asked to monitor these behaviors separately, but she often reported one number and did not indicate what percent was picking and what percent was pulling. Thus, partly out of necessity, and partly because she reported them being functionally the same, they are reported as one behavior class. On the days that she did report the pulling and picking separately, 66\% was skin picking, and there were no days were it was only hair pulling, although some days she only skin picked.

Assessments of comorbid psychological conditions were not formally conducted, but Participant 4 had received a diagnosis of major depression from a previous psychologist. Her score on the Beck Depression Inventory-II supported this diagnosis (see Table 2).

\section{Measures}

\section{Skin Picking Severity}

Self-monitoring (Twohig \& Woods, 2004). Participants were given “3 x 5” note cards and asked to place a mark on the card each time they picked their skin. Skin picking was operationally defined as “using one’s fingers or an instrument to attempt or actually remove a 
piece of skin.” Clients were instructed to record each picking attempt, thus, one bout of picking could include many picks. At the end of each day, the participants reported the number of picks to the experimenter via telephone to a message machine.

Objective skin-damage ratings (Twohig \& Woods, 2001). In order to validate self-reports of picking, pretreatment, post-treatment, and three-month follow-up photographs were taken approximately $30 \mathrm{~cm}$ from the participant's picking area with a digital camera. Five professionals in clinical psychology, blind to the participants' phases in the study, initially viewed all three photographs of each participant to see the range of each participant's damage. Next, the pictures were randomly presented, and for each picture, the raters were asked to rate “how damaged does the skin appear to be.” This was rated on a seven point Likert-type scale where 1 = not damaged at all and 7 = very damaged. Mean pre-treatment, post-treatment, and follow-up scores were calculated for each participant at each point in the study.

Skin Picking Impact Scale (SPIS, Keuthen et al., 2001). The SPIS is a 10-question selfreport measure that assesses the severity of skin picking. Each question is rated on a 0 to 5 point scale, with higher scores representing greater picking severity. The SPIS is scored by summing the scores from the individual items. Scores on the SPIS range from 0 to 50, and a score of 7 or above indicates clinical skin picking. The SPIS has demonstrated good internal consistency $(\alpha=$ 0.88 to 0.93 ) with whole part correlations for the 10 items being between 0.60 and 0.80 , high reliability, and acceptable convergent and divergent validity when compared to measures of psychological functioning (Keuthen et al., 2001).

Massachusetts General Hospital Hairpulling Scale (MGH-HS; Keuthen et al., 1995). The MGH-HS is a seven-question self-report measure of hair pulling severity. Each question is answered on a 0 to 4 point scale, with higher scores indicating greater pulling severity. The 
MGH-HS is scored by summing the scores from the individual items. Scores on the MGH-HS range from 0 to 28. The MGH-HS has demonstrated adequate internal consistency $\alpha=.89$ (Keuthen et al., 1995), good test-retest reliability $r=.97$, acceptable convergent and divergent validity when compared to measures of psychological functioning, and has been found to be sensitive to change when correlated with other measures of TTM (O’Sullivan et al., 1995).

\section{General Psychological Functioning}

Acceptance and Action Questionnaire (AAQ; Hayes et al., 2004). The AAQ is a 9-item questionnaire that measures experiential avoidance broadly conceived (items target a range of ACT-relevant processes that are thought to contribute to psychological inflexibility including willingness, lack of action, and cognitive fusion). Questions are rated on a seven-point Likerttype scale. Lower scores reflect greater experiential willingness and ability to act in the presence of difficult thoughts and feelings. The AAQ has been found to have adequate internal consistency and good convergent and discriminant validity (Hayes et al., 2004).

Beck Anxiety Inventory (BAI; Beck, Epstein, Brown, \& Steer, 1988). The BAI is a 21question self-report measure that assesses anxiety. The BAI has high internal consistency $(\alpha<$ $.90)$, adequate test-retest reliability $(r>.60)$, and moderate to high convergent and discriminant validity.

Beck Depression Inventory-II (BDI-II; Beck, 1996). The BDI-II is a self-report measure that assesses the severity of depression. The BDI-II has shown good test-retest reliability ( $r=$ .93), and has demonstrated a high correlation with the original BDI ( $r=.93$; Beck, 1996).

Treatment Evaluation Inventory-Short Form (TEI-SF; Kelley, Heffer, Gresham, \& Elliot, 1989). The TEI-SF measures the participant's opinion of treatment. A modified version of the TEI-SF, which contained seven questions instead of nine, was used in this study (also used by 
Twohig \& Woods, 2004). The two questions that were removed concerned developmental disabilities and were considered not appropriate for this population. Each of the questions was rated on a 5-point Likert-type scale with higher numbers reflecting greater acceptability. The values were summed and resulted in a treatment acceptability score for each participant. Scores over 21 indicate greater acceptability than unacceptability of the intervention. The original TEISF instrument has a reliable factor structure and is internally consistent, $\alpha=.85$.

\section{Procedure}

During the initial assessment session, skin picking was assessed using a clinical interview, and its severity was assessed using the SPIS. Relevant demographic data were collected, a photograph of the damaged area was taken, and participants completed questionnaire measures. Finally, participants were given index cards to self-monitor the number of skin picks per day, throughout the course of the study.

The intervention was evaluated in one non-concurrent (Participants 1, 2, and 3) and one concurrent multiple baseline design (Participants 4 and 5). Treatment consisted of eight, weekly, one-hour sessions of ACT for skin picking based heavily on the ACT manual (Hayes et al., 1999). The first author served as the therapist for all participants. The second author, a developer of the treatment, trained the first author. All sessions were videotaped, and 25\% (at least one randomly selected tape of each session) were scored for treatment integrity by the third author.

Participants 1, 2, and 4 began treatment after adequate baselines for assessing level, trend, and variability were collected. Because Participant 1 never reached zero levels, Participant 3 began treatment after a clear change had been seen in Participant 2 (this was non-concurrent). Participant 5 began treatment only after Participant 4 concurrently began to show changes to near zero levels. 
Acceptance and Commitment Therapy. A journal-length article does not provide the space to fully describe ACT as it is applied to skin picking. In this section, we will briefly describe the intervention and provide the references to a fuller description. The complete manual is available from the first author or on the ACT website (www.acceptanceandcommitmenttherapy.com).

All sessions followed the same pattern: events since the last session and homework were reviewed, the material from the previous session was reviewed and new material was presented, and new homework was assigned and behavioral commitment exercises were agreed upon. Behavioral commitment exercises generally involved committing to not picking for specified periods of time, or staying below specified levels. During behavioral commitment exercises, clients were instructed to practice the procedures that they were taught in session.

Treatment began by collecting pertinent information on the participant's skin picking, introducing the treatment, and forming a verbal contract for the eight sessions. ACT for skin picking formally began by distinguishing the difference between the urges to pick (i.e., thoughts or feelings that function as establishing operations for picking) and the actual picking. Next, the participant was asked what he or she has been trying to do to decrease the urge to pick, what has worked, and what has not. This illustrated that the only way to get rid of the urge was to skin pick, but that only worked for brief periods of time. The "Person in the Hole" metaphor (Hayes et al., 1999, p. 101) was used to demonstrate the ultimate ineffectiveness of attempts to eliminate one’s urges to skin pick.

Sessions three and four generally focused on illustrating how attempts to control the urge to pick might be the problem rather than the solution. These sessions involved exercises aimed at illustrating the limitations of control when aimed at private events, such as trying not think of something, such as chocolate cake (Hayes et al., 1999, p. 124) or trying to not get nervous, while 
hooked to a polygraph (Hayes et al., 1999, p. 123). These exercises were designed to help the participant experience the difference between urges to skin pick (and uncontrollable private event) and picking (a controllable public event), hopefully shifting the focus from decreasing the urge to pick to decreasing the picking. The “Two Scales Metaphor” (Hayes et al., 1999, p.133) further illustrated this approach.

Sessions five and six focused on changing the psychological function of the urge to pick from something threatening to just another verbal event. This involved defusion exercises (repeating a thought over and over until it loses it's meaning, Masuda, Hayes, Sackett, \& Twohig, 2004, and Passengers on the Bus metaphor, Hayes et al. 1999, p. 157), contact with the present moment or mindfulness exercises (e.g., Soldiers In The Parade Exercise, Hayes et al., 1999, p. 158), and “self as context” work (e.g., Chessboard Metaphor, Hayes et al., 1999, p. 190).

The final two sessions, sessions seven and eight, involved reviewing the material from the earlier sessions, values work (following ones values rather than working to decreased private events), and discussions regarding maintenance of ones decreases in picking.

One week after treatment was completed, the participants were asked to discontinue selfmonitoring, and return to the clinic for the post-treatment assessment, which involved completing the AAQ, BAI, BDI-II, SPIS, and TEI-SF. Another picture was taken of the damaged area. At three months post-treatment, the participants were asked to self-monitor for two additional concurrent days, complete the same assessments completed at post-treatment (with the exception of the TEI-SF), and another photograph was taken.

Treatment Integrity. The tapes were scored for the quantity and quality of the coverage of each component of ACT using a validated and reliable scoring system previously used in ACT 
research (Twohig et al., in press). Scores of 1 indicate the variable was never explicitly covered, $2=$ the variable occurred at least once and not in an in-depth manner, $3=$ the variable occurred several times and was covered at least once in a moderately in-depth manner, $4=$ the variable occurred with relatively high frequency and was addressed in a moderately in depth manner, 5 = the variable occurred with high frequency and was covered in a very in-depth manner. Means for each component over the eight sessions are as follows: creative hopelessness / workability = 3.8 (range 2-5), willingness / acceptance $=3.8$ (range 1-5), defusion $=2.3$ (range 1-3), values $=1.6$ (range1-3), committed action = 1.6 (range 1-4), and general assessment of participant's functioning = 3.1 (range 2-4). The therapist's overall adherence to the manual and overall competence received high ratings, $M=4.4$ (range 4-5) and 3.9 (range 3-5) respectively. In addition, the sessions were scored for therapeutic practices that were inconsistent with ACT including challenging cognitive content, promoting change strategies that involved avoidance of the urge to pick, indicating that thoughts or feelings cause overt behavior, and use of cognitive therapy rationales. Because this specific protocol was deliberately limited so as to avoid the use of any SHR components, awareness training and competing response training were also scored for their occurrence. All ACT inconsistent measures and the SHR measure received scores of one, indicating they were not observed.

\section{Results}

\section{Skin Picking Frequency}

Self-monitoring data for all participants are presented in Figure 1. None of the participants showed decreases in self-reported skin picking during a one to seven week baseline. Four of the five showed large reductions during treatment, but these gains were only fully maintained for one of the four participants at three-month follow-up. Data were collected throughout, but post- 
treatment is considered data collected after the eighth (i.e., final) session. All self-report measure findings are reported in Figure 2.

Participant 1. Participant 1's baseline level of picking was $M=57.5(S D=35.7)$. She showed decreases in picking while receiving treatment, but never stopped picking (posttreatment $M=19.3, S D=19.7)$. She had high levels of picking at follow-up $(M=100, S D=$ 14.1).

Participant 2. Pre-treatment level for participant 2's skin picking and hair pulling was $M=$ $24.5(S D=24.1)$. Her picking/pulling reached near zero levels after the fourth session, and had completely stopped picking/pulling by post-treatment $(M=0, S D=0)$. Her picking/pulling returned at follow-up $(M=16, S D=8.5)$, although it was still below baseline levels.

Participant 3. Participant 3 reported skin picking 118.5 times per day on average $(S D=$ 42.3) during baseline. She showed a dramatic decrease in her picking upon beginning treatment, and reached very low levels by post-treatment (post-treatment $M=5.60, S D=3$ ). No picking was reported at follow-up $(M=0, S D=0)$.

Participant 4. Participant 4's baseline mean was $166.3(S D=49.2)$. She showed a dramatic decrease in her picking upon implementation of the treatment (post-treatment $M=4.7$, $S D=4.3)$. Her picking returned to near baseline levels at follow-up $(M=120, S D=56.6)$.

Participant 5. Participant 5 reported skin picking 95.7 times per day on average during pre-treatment $(\mathrm{SD}=45.4)$. She showed a very gradual decrease in her picking and was able to reach very low levels of picking by her sixth session (post-treatment $\mathrm{M}=3.4$, SD = 3.3). Her picking returned at follow-up, but it was below baseline levels $(M=56, \mathrm{SD}=17)$. 


\section{Collateral Measures of Skin Picking Severity}

Table 2 shows the specific scores for all participants on the SPIS and on all other selfreport instruments, including the BAI, BDI-II, AAQ, MGH hair pulling scale, and treatment acceptability measures. The findings on the SPIS mirrored the clients' self-reports of skin picking. Pre-treatment, post-treatment, and follow-up means were $24.8(S D=15.3), 12.6(S D=$ 11.5), and $19.8(S D=16.3)$ respectively (see Table 2 for exact scores). Photograph ratings also generally corroborated the self-reports of the participants. Participants, 2, 3, 4, and 5's pretreatment photographs were rated as having the most damage, with substantial decreases at posttreatment, and then slight increases in damage at follow-up. These ratings are consistent with the self-reports except for Participant 3 who did not report any picking at follow-up but showed a slight increase in apparent damage. Participant 1's ratings were inconsistent with her self-report; this might be the result of her continuing to pick throughout treatment. Her initial damage was also rated very low.

\section{Anxiety and Depression}

All participants completed the BAI and BDI-II at pre-treatment, post-treatment, and follow-up. Participant 4 was clinically elevated on her depression throughout the study. She was also in the severe range for anxiety at pre-treatment for anxiety; in the mild range at posttreatment, and in the moderate range at FU. All other participants started with very low scores on both measures, and showed modest reductions from pre to post-treatment with gains generally being maintained at follow-up (see Table 2 for exact scores).

\section{Treatment Process Measures}

All participants completed the AAQ at pre-treatment, post-treatment, and follow-up. Three of the five participants showed large reductions from pre to post-treatment with continued 
or maintained gains at follow-up. Pre-treatment, post-treatment, and follow-up means were 24.8 $(S D=5.6), 18(S D=10.6)$, and $16.8(S D=8.6)$ respectively (see Table 2 for exact scores).

\section{Treatment Acceptability Data}

All participants completed the TEI-SF at post-treatment and all participants rated the treatment as acceptable (see Table 2 for exact scores).

\section{Discussion}

To date there are only two other controlled studies that evaluated psychological treatments for skin picking (Teng et al., in press; Twohig \& Woods, 2001) and both evaluated SHR. This present study cannot be directly compared to these studies because the very small numbers do not allow it - rather the present study was designed to assess whether a different yet compatible behavioral approach could also positively impact the problem of skin picking. The data suggest that it can, but that additional treatment development is needed, particularly in the maintenance of gains.

Self-monitoring of skin picking showed that four of the five participants reached near zero levels of picking by post-treatment, with gains being maintained for one of the four participants at follow-up. Patterns on the self-reports of skin picking were generally corroborated by objective ratings of photographs of skin picking damage. Reductions were found from pre to post and pre to follow-up on the SPIS, and on a general measure of anxiety. At the process level, reductions were found on a measure of experiential avoidance. All participants rated the intervention as socially acceptable.

Methodologically, this study is limited by its use of self-report data, but this limitation was reduced through social validity ratings of actual pictures of damage. The photograph ratings did not exactly match the self-report of skin picking for all participants, particularly at follow-up 
where there was a very small period of self-monitoring. Ratings of skin damage do not always correspond with picking frequency, however, because even a limited number of picks can cause a great deal of damage and frequent minimal picks can cause less severe damage. The objective ratings of damage did seem to comport with the general conclusions being drawn from selfmonitoring data.

This study was not designed to rule out the effects of nonspecific variables that might have affected the treatment outcome. Future studies need to employ control conditions or groups to address these issues and to apply them to a more diverse sample than the Caucasian female participants used in the present study.

The biggest focus for future development is the maintenance of gains. The present study deliberately used a stripped down ACT protocol for scientific reasons but the most obvious next step is to combine ACT with more traditional behavior modification procedures. This is recommended within the ACT model itself: "During the latter portions ... ACT takes on the character of traditional behavior therapy, and virtually any behavior change technique is acceptable. The difference is that behavior change goals, guided exposure, social skills training, modeling, role playing, couples work, and so on are integrated with an ACT perspective. Behavior change is a kind of willingness exercise, linked to chosen values” (Hayes et al., 1999, p. 258). Consistent with this approach previous, ACT studies for trichotillomania have incorporated SHR and have been shown to be effective (Twohig \& Woods, 2004, Woods et al., in press). Other possibilities include structured mindful picking, scheduled and tapered picking, cue exposure, or other such methods. These combinations then need to be compared to their component elements alone as part of an inductive technique building strategy. 
The present study asked whether ACT has potential as a method for the treatment of chronic skin picking. The results suggest that it does but that additional treatment development is needed. 


\section{References}

American Psychiatric Association (2000). Diagnostic and statistical manual of mental disorders (4th ed. Text Revision). Washington, DC: Author.

Beck, A. T. (1996). Beck Depression Inventory ( $2^{\text {nd }}$ ed.). San Antonio, TX: The Psychological Corporation.

Beck, A. T., Epstein, N. Brown, G., \& Steer, R. A. (1988). An inventory for measuring clinical anxiety: Psychometric properties. Journal of Consulting and Clinical Psychology, 56, 893-897.

Begotka, A. M., Woods, D. W., \& Wetterneck, C. T. (2004). The relationship between experiential avoidance and the severity of trichotillomania in a nonreferred sample. Journal of Behavior Therapy and Experimental Psychiatry, 35, 17-24.

Bohne, A., Wilhelm, S., Keuthen, N. J., Baer, L., \& Jenike, M. A. (2002). Skin picking in German students: Prevalence, Phenomenology, and associated characteristics. Behavior Modification, 26, 320-339.

Christenson, G. A. \& Mansueto, C. S. (1999). Trichotillomania: Descriptive characteristics and phenomenology. In D. J. Stein, G. A. Christenson, \& E. Hollander (Eds.). Trichotillomania. (1-32). Washington DC: American Psychiatric Press.

du Toit, P. L., van Kradenburg, J., Niehaus, D. J. H., \& Stein, D. J. (2001). Characteristics and phenomenology of hair-pulling: An exploration of subtypes. Comprehensive Psychiatry, 42, 247-256.

Hayes, S. C., Strosahl, K. D., \& Wilson, K. G. (1999). Acceptance and Commitment Therapy: An experiential approach to behavior change. New York: Guilford Press. 
Hayes, S. C., Strosahl, K. D., Wilson, K. G., Bissett, R. T., Pistorello, J., Toarmino, et al. (2004). Measuring experiential avoidance: A preliminary test of a working model. The Psychological Record, 54, 553-578.

Keuthen, N. J., Deckersbach, T., Wilhelm, S. Engelhard, I., Forker, A., O’Sullivan, R. L. Jenike, M. A., \& Baer, M. (2001). The skin picking impact scale (SPIS): Scale development and psychometric analyses. Psychosomatics: Journal of Consultation Liaison Psychiatry, 42, 397-403.

Keuthen, N. J., O’Sullivan, R. L., Ricciardi, J. N., Shera, D., Savage, C. A., Borgmann, A. S., Jenike, M. A., \& Baer, L. (1995). The Massachusetts General Hospital (MGH) hair pulling scale: I. Development and factor analyses. Psychotherapy and Psychosomatics, $64,141-145$.

Kelly, M. L., Heffer, R. W., Gresham, F. M., \& Elliot, S. N. (1989). Development of a modified treatment evaluation inventory. Journal of Psychopathology and Behavioral Assessment, $11,235-247$.

Masuda, A., Hayes, S. C., Sackett, C. F., \& Twohig, M. P. (2004). Cognitive defusion and selfrelevant negative thoughts: Examining the impact of a ninety year old technique. Behaviour Research and Therapy, 42, 477-485.

O’Sullivan, R. L., Keuthen, N. J., Hayday, C. F., Ricciardi, J. N., Buttolph, M. L., Jenike, M. A., \& Baer, L. (1995) The Massachusetts General Hospital (MGH) hair pulling scale: Reliability and validity. Psychotherapy and Psychosomatics, 64, 146-148.

O’Sullivan, R. L. Phillips, K. A., Keuthen, N. J., \& Wilhelm, S. (1998). Near fatal skin picking from delusional body dysmorphic disorder responsive to fluvoxamine. Psychosomatics, 39, $1-4$ 
Teng, E. J., Woods, D. W., \& Twohig, M. P. (in press). Habit reversal as a treatment for chronic skin picking. Behavior Modification.

Teng, E. T., Woods, D. W., Twohig, M. P., \& Marcks, B. A. (2002). Body focused repetitive behavior problems: Prevalence in a non-referred population and differences in somatic awareness. Behavior Modification, 26, 340-360.

Twohig, M. P., Hayes, S. C. \& Masuda, A. (in press). Increasing willingness to experience obsessions: Acceptance and Commitment Therapy as a treatment for Obsessive Compulsive Disorder. Behavior Therapy.

Twohig, M. P., \& Woods, D. W. (2001). Habit Reversal as a treatment for chronic skin picking in typically developing adult male siblings. Journal of Applied Behavior Analysis. 34, 217-220.

Twohig, M. P., \& Woods, D. W. (2004). A preliminary investigation of acceptance and commitment therapy and habit reversal as a treatment for trichotillomania. Behavior Therapy, 35, 803-820.

Woods, D. W., Wetterneck, C. T., Flessner, C. A. (in press). A controlled evaluation of Acceptance and Commitment Therapy plus Habit Reversal as a treatment for trichotillomania. Behaviour Research and Therapy. 


\section{Author's Note}

Michael P. Twohig, Steven C. Hayes, Akihiko Masuda are in the Department of Psychology / 298, University of Nevada, Reno, NV 89557-0062.

A copy of the complete treatment manual is available from the first author, Michael Twohig (e-mail: twohigm@unr.nevada.edu) and is posted on the ACT website: www.acceptanceandcommitmenttherapy.com. 
Table 1

Participant characteristics table

\begin{tabular}{|c|c|c|c|c|c|}
\hline Participant & $\mathrm{P} 1$ & $\mathrm{P} 2$ & P3 & $\mathrm{P} 4$ & P5 \\
\hline Mar. Stat. & $\mathrm{S}$ & $\mathrm{S}$ & $\mathrm{S}$ & $\mathrm{S}$ & M \\
\hline Age & 23 & 28 & 19 & 21 & 24 \\
\hline Yrs of Ed. & 15 & 14 & 13 & 15 & 14 \\
\hline Yrs. Picking & 19 & 14 & 15 & 13 & 14 \\
\hline Yrs. Pulling & & 18 & & & \\
\hline Picking site & fingers & arms & fingers & fingers & fingers \\
\hline Pulling site & & scalp & & & \\
\hline Previous tx. & EMDR & none & $\begin{array}{l}\text { band-aids } \\
\text { gloves }\end{array}$ & $\begin{array}{l}\text { band-aids } \\
\text { tape } \\
\text { aversive ts }\end{array}$ & $\begin{array}{l}\text { band-aids } \\
\text { aversive tastes }\end{array}$ \\
\hline
\end{tabular}

Marital status, $\mathrm{M}=$ married, $\mathrm{S}$ = single; Years of education begin with first grade (i.e., $12=$ high school education, 16 = four years of post high school education); Previous treatments EMDR = eye movement desensitization and reprocessing, gloves $=$ wear gloves over hands, band-aid $=$ put band-aid over picking site, tape = put tape over picking site, aversive taste $=$ put aversive tasting substance on picking site 
Table 2.

Assessment scores for pre-treatment, post-treatment, and three-month follow-up

\begin{tabular}{|c|c|c|c|c|c|c|c|c|c|}
\hline \multirow[t]{2}{*}{ Participant } & \multirow[b]{2}{*}{ pre } & & \multicolumn{3}{|c|}{ P2 } & \multicolumn{3}{|c|}{ P3 } \\
\hline & & post & FU & pre & post & FU & pre & post & FU \\
\hline AAQ & 18 & 9 & 9 & 21 & 15 & 18 & 24 & 10 & 9 \\
\hline BAI & 9 & 2 & 3 & 15 & 4 & 5 & 7 & 0 & 2 \\
\hline BDI-II & 2 & 2 & 2 & 18 & 2 & 9 & 4 & 0 & 0 \\
\hline SPIS & 15 & 18 & 17 & 45 & 29 & 43 & 10 & 0 & 1 \\
\hline MGH - HS & & & & 17 & 10 & 7 & & & \\
\hline TEI-SF & & 29 & & & 26 & & & 26 & \\
\hline Participant & Pre & $\begin{array}{l}\text { P4 } \\
\text { post }\end{array}$ & FU & pre & $\begin{array}{l}\text { P5 } \\
\text { post }\end{array}$ & FU & & & \\
\hline AAQ & 30 & 35 & 30 & 31 & 21 & 18 & & & \\
\hline BAI & 31 & 19 & 21 & 8 & 3 & 5 & & & \\
\hline BDI & 29 & 35 & 34 & 8 & 3 & 5 & & & \\
\hline SPIS & 37 & 12 & 28 & 17 & 4 & 10 & & & \\
\hline TEI-SF & & 26 & & & 27 & & & & \\
\hline
\end{tabular}

$\overline{\mathrm{AAQ}}=$ Acceptance and Action Questionnaire; BAI = Beck Anxiety Inventory; BDI-II = Beck Depression Inventory-II; SPIS = Skin Picking Impact Scale; MGH - HS = Massachusetts General Hospital Hairpulling Scale; TEI-SF = Treatment Evaluation Inventory - Short Form 


\section{Figure Captions}

Figure 1. Frequency of self-monitored picking across all participants.

Figure 2. Rated skin damage for all participants at pre-treatment, post-treatment, and follow-up. 


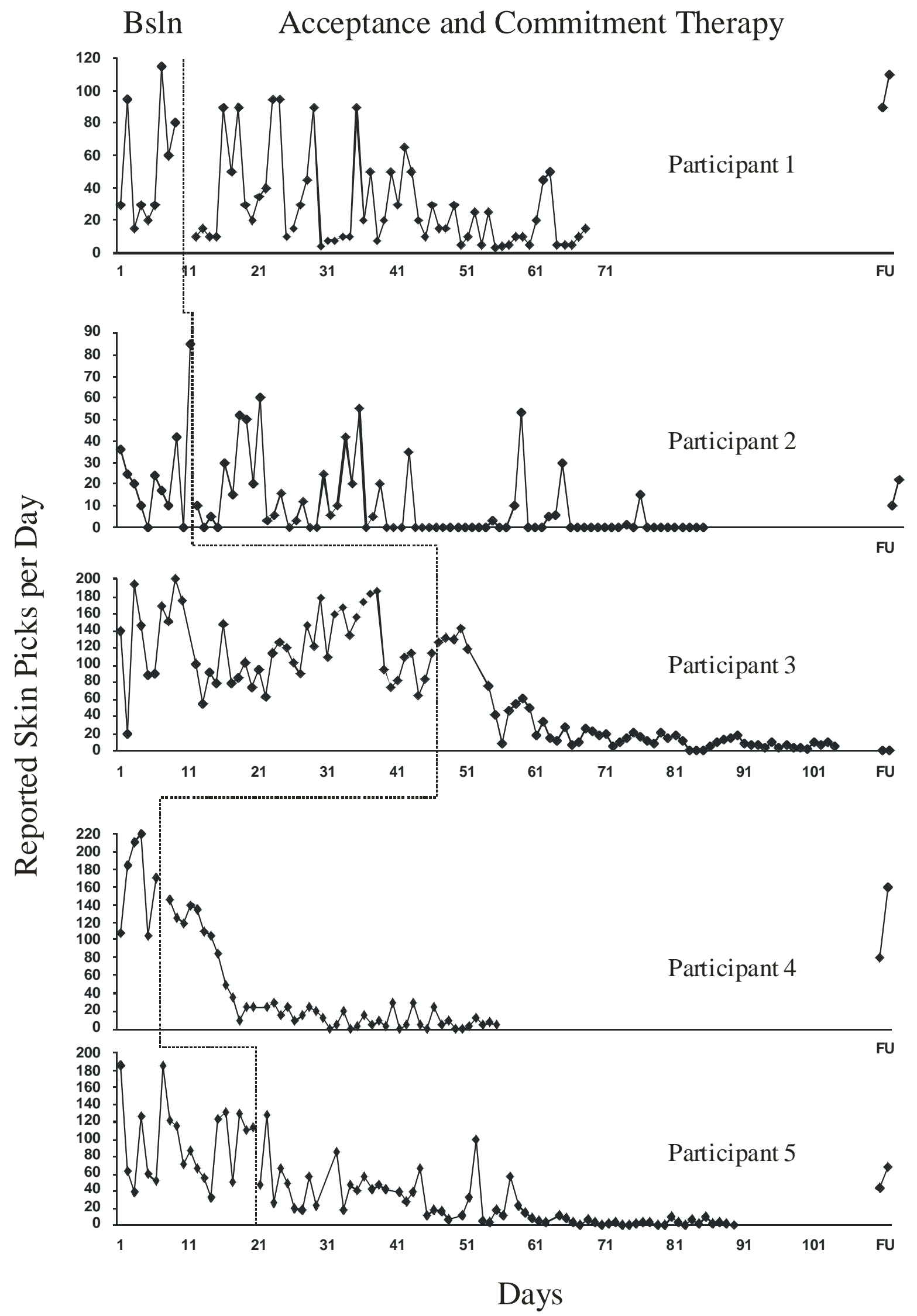


ACT for Skin Picking 26

\section{Rated Skin Damage}

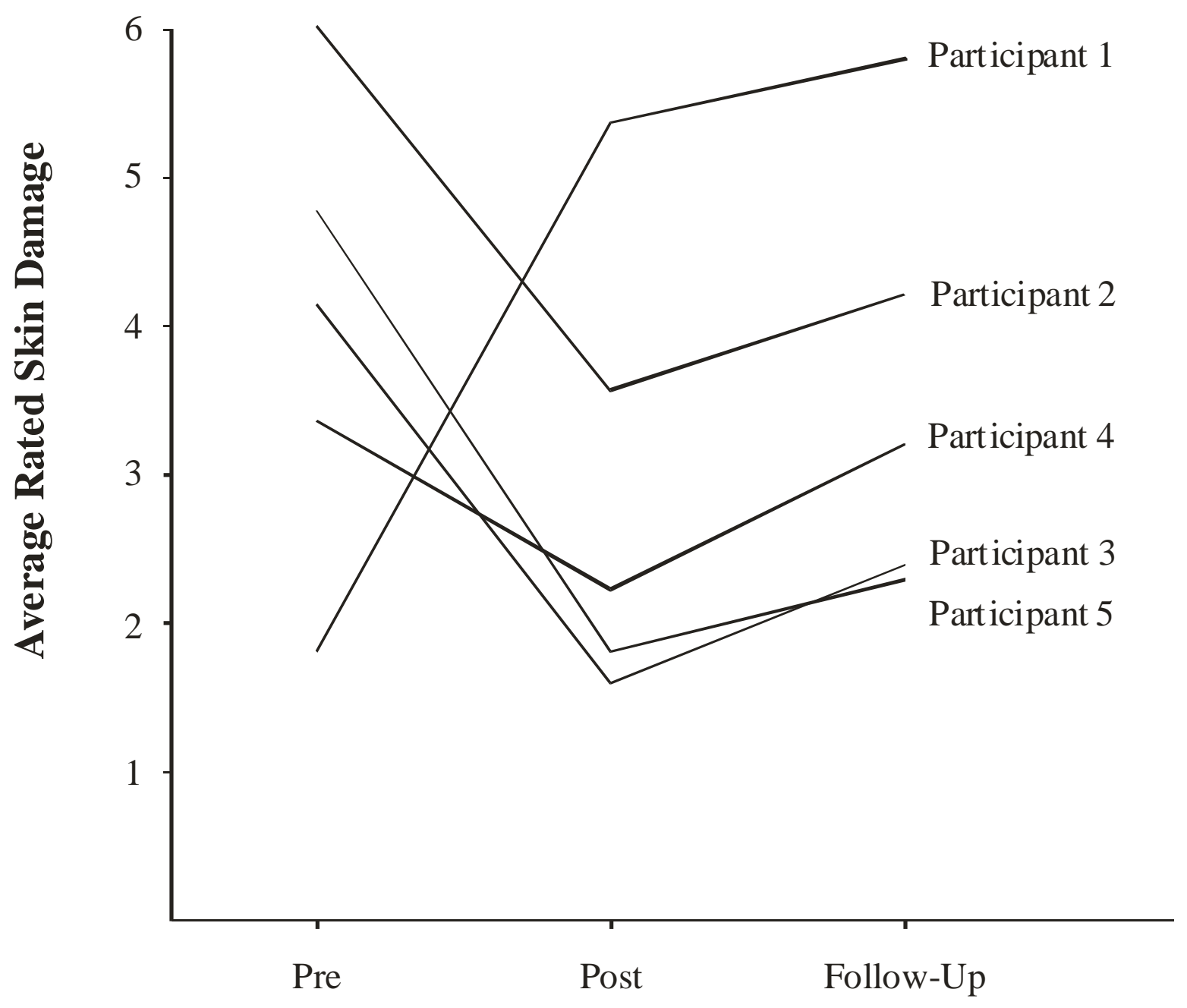

Phase 